\title{
Proteotoxicity in the endoplasmic reticulum: lessons from the Akita diabetic mouse
}

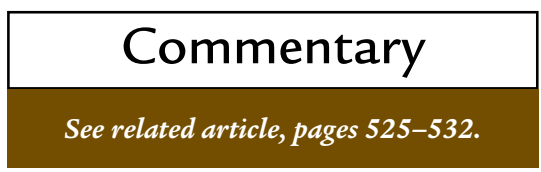

\author{
David Ron \\ Skirball Institute of Biomolecular Medicine, Departments of Medicine and Cell Biology, \\ New York University School of Medicine, New York, New York, USA \\ Address correspondence to: David Ron, Skirball Institute 3-10, 540 First Avenue, New York, New York 10016, USA. \\ Phone: (212) 263-7786; Fax: (212) 263-8951; E-mail: ron@saturn.med.nyu.edu.
}

J. Clin. Invest. 109:443-445 (2002). DOI:10.1172/JCI200215020.

Mutations that impair polypeptide folding commonly result in an unstable and hypofunctional gene product. Their phenotype reflects this loss of function, and the resulting genetic disorder is usually transmitted as a recessive trait. Common forms of cystic fibrosis, hemophilia, and familial hypercholesterolemia are examples of this genetic mechanism in action. A second class of mutations (so-called gain-of-function mutations) encodes proteins with new functions, whose associated disorders are typically transmitted as dominant traits. Some of these are "dominant negative" alleles whose encoded protein can interact with components of the cellular machinery normally accessed by the wild-type gene product. With varying degrees of specificity, these mutations affect the activities of the wild-type allele, for example by disrupting the assembly of a multisubunit complex. Recent observations suggest that gainof-function mutations that affect protein folding can also impair cellular function by less specific mechanisms related to the ability of the mutant protein to challenge the folding capacity in specific cellular compartments. Such mutant proteins are hypothesized to act as proteotoxins and may play a role in important human diseases $(1,2)$. The paper by Oyadomari et al. (3) appearing in this issue of the JCI addresses important issues related to proteotoxicity in the endoplasmic reticulum (ER).

The Ins $2^{\mathrm{C} 96 \mathrm{Y}}$ mutation found in the Akita diabetic mouse precludes formation of an essential disulfide bond between insulin 2 chains and prevents proper folding and processing of this protein. The mutant, malfolded proinsulin-2 is retained in the pancreatic $\beta$ cell ER (4), presumably by the quality control mechanisms that normally allow only properly folded proteins to exit the ER and progress in the secretory pathway (5). Mice carrying the Ins2 ${ }^{C 96 Y}$ mutation develop progressive diabetes mellitus, a phenotype that probably reflects more than merely loss of hormone production by the mutant Ins 2 allele, as rodents have two insulin genes (Ins1 and Ins2) and even loss of both copies of Ins 2 is fully compensated (6).

What, then, is the basis of the gainof-function phenotype associated with the Ins $2^{\mathrm{C} 96 \mathrm{Y}}$ Akita mutation? Ins $2^{\mathrm{C} 96 \mathrm{Y}}$ mutant mice are born with normalsized islets of Langerhans and a normal complement of insulin-producing $\beta$ cells. Over time, however, they undergo a progressive loss of $\beta$ cells. Apoptosis of these cells correlates with the development of diabetes mellitus. This aspect of the pathophysiology of the Akita mouse can be reproduced in vitro by expressing high levels of Ins2 ${ }^{\mathrm{C} 96 Y}$ (but not wild-type Ins2) in the $\beta$ cell line Min6 (3). It is likely, therefore, that expression of Ins $2^{C 96 Y}$ is toxic to islet cells and that loss of $\beta$ cell mass plays a role in the development of hyperglycemia in the Akita mouse.

\section{ER stress and cell death}

Eukaryotic cells maintain equilibrium between the load of client proteins their ER must process and the capacity of the organelle to carry out this function. A threat to this equilibrium is referred to as ER stress and is counteracted by two distinct adaptations collectively referred to as the unfolded protein response (UPR) $(7,8)$. The first of these adaptations attenuates protein biosynthesis, to immediately relieve the load on the organelle. The second increases the synthesis of components of the machinery by which the ER processes client proteins (upregulating chaperones, glycosylation enzymes, oxidases, and other ER resident proteins). Several mediators of UPR signaling have been identified recently. Their activity is consistent with a model whereby the cell tries to defend a certain chaperone reserve. When this functional reserve is challenged, it appears, the cell senses ER stress and responds by activating the $\operatorname{UPR}(9,10)$. Accordingly, the level of chaperones and other ER components is a measure of the level of ER stress the cell is under.

High levels of sustained ER stress can also lead to programmed cell death, and some of the mediators of this response have recently been identified. One, caspase-12, an ER-associated cell death effector, is specifically activated by proteolytic processing during ER stress. Another, the transcription factor $\mathrm{CHOP}$, is strongly upregulated during ER stress. Mutations in either of the corresponding genes inhibit cell death caused by ER stress without otherwise affecting the $\operatorname{UPR}(11,12)$. IRE1, one of the direct mediators of the UPR, activates Jun $\mathrm{N}$-terminal kinase (JNK) and can also promote cell death $(13,14)$. It appears, therefore, that cell death during ER stress results from the activity of dedicated components.

It had been noted earlier that the islet cells in the Akita mice express higher levels of the ER chaperone BiP, a marker of ER stress (4). Now, Oyadomari and colleagues show that $\mathrm{CHOP}$ is also activated in the Akita mouse islets (3). Neither of these findings is particularly surprising, given the impact of the Ins $2^{\mathrm{C} 96 \mathrm{Y}}$ mutation on insulin 2 folding. Insulin is a major ER client protein in $\beta$ cells, and one might imagine that expression of high levels 
Table 1

Diseases potentially associated with ER proteotoxicity

Disease
Pelizaeus-Merzbacher leukodystrophy
Charcot-Marie-Tooth disease
Hereditary neurogenic diabetes insipidus
Pre-senile dementia/Myoclonus
$\alpha 1$-Antitrypsin deficiency
Osteogenesis imperfecta

Mutant protein
PLP
PMP22, P0
AVP
Neuroserpin
Q1-Antitrypsin
Collagen type I

Cell affected
Oligodendrocyte
Schwann cell
AVP neuron
Cortical neuron
Hepatocyte
Osteoblast

Reference

25

26

27

28

29

30

In each of the diseases listed in this table, a gain of function mutation that impairs folding of a highly expressed ER client protein adversely affects ER function, causing ER stress in the cell expressing the mutant protein. While loss of wild-type gene function may play an important role in some of these disorders, proteotoxicity in the ER is proposed to be a common feature in their pathogenesis.

of a mutant insulin protein could severely tax the folding capacity of the organelle and elicit ER stress. The crucial question in this system is whether ER stress contributes to $\beta$ cell death and the development of diabetes mellitus. A positive answer could not be taken for granted, as ER stress is a physiological phenomenon in many cells (including $\beta$ cells) and can be well compensated for by physiological adaptations (15). Furthermore, there are other plausible reasons for islet cell death and diabetes mellitus in the Akita mouse. For example, the mutant insulin may impair the processing or secretion of wild-type insulin, by specific dominant negative mechanisms.

To examine the role of ER stress in islet cell death and diabetes mellitus, Oyadomari and colleagues (3) bred the Akita mutation onto the $\mathrm{CHOP}$ knockout background. The $\mathrm{CHOP}$ gene is induced by ER stress through a signaling pathway that involves activation of the pancreas ER kinase (PERK) and phosphorylation of the translation initiation factor eIF $2 \alpha$. CHOP encodes a transcription factor that promotes programmed cell death $(11,16)$, and the authors reasoned that if ER stress-mediated cell death plays a role in the phenotype of the Akita mouse, a background mutation in CHOP could ameliorate its features. This is exactly what they observed: $\mathrm{CHOP}^{-/-}$;Ins $2^{\mathrm{C} 96 \mathrm{Y} / W \mathrm{~T}}$ mice had preserved islet cell mass, less $\beta$ cell apoptosis, delayed onset of hyperglycemia, higher pancreatic insulin content, and greater body weight than $\mathrm{CHOP}^{-/+}$; Ins2 ${ }^{\mathrm{C} 96 \mathrm{Y} / \mathrm{WT}}$ or $\mathrm{CHOP}^{+/+}$;Ins2 $2^{\mathrm{C} 96 \mathrm{Y} / \mathrm{WT}}$ mice. Because CHOP activation is a nonspecific feature of ER stress, these results suggest that the mutant insulin 2 protein accumulating in the ER of the $\beta$ cell exerts at least some of its effects as a nonspecific proteotoxin.

While CHOP is strongly activated by ER stress, other stress signals such as oxidative stress and amino acid deprivation that bypass the ER altogether can also induce $C H O P$ gene expression $(17,18)$; therefore it is formally possible that the contribution of $C H O P$ to death of the Akita $\beta$ cells also reflects the activity of other stress pathways operating in these cells. However, the role of $C H O P$ in promoting cell death in response to these other forms of stress remains unproven. It is also important to note that the CHOP mutation provided no measurable benefit to the severely affected homozygous Ins $2^{\text {C96Y/C96Y mice. This }}$ last observation suggests the existence of $\mathrm{CHOP}$-independent ER stress-mediated cell death pathways or other unrelated mechanisms causing dysfunction of the homozygous mutant $\beta$ cells.

The aforementioned caveats aside, it is interesting to relate the observations described here to the possible role of nonspecific ER proteotoxicity in the pathogenesis of other genetic diseases and perhaps in other forms of diabetes mellitus. There are several human diseases caused by gain-of-function mutations affecting the folding of abundantly expressed ER client proteins (Table 1). Death or dysfunction of cells expressing the mutant protein goes a long way toward explaining the phenotype in these ER stress-associated genetic disorders, whereas interference of the mutant allele with the function of the wild-type one may be less important. In some cases, such as the PLP gene associated with Pelizaeus-Merzbacher leukodystrophy, or the PMP22 and P0 genes associated with Charcot-Marie-Tooth disease, the null state has a very different and sometimes weaker phenotype than does the dominant mutation affecting protein folding $(19,20)$. These findings are consistent with the idea that cell dysfunction or death by mechanisms related to those described here by Oyadomari and colleagues (3) might play a role in the pathogenesis of important human diseases.

Loss of $\beta$ cell function plays an important role in the development of type 2 diabetes mellitus, but its causes remain unknown. Insulin biosynthesis is likely to result in a significant physiological load on the $\beta$ cell. This load is certainly increased by insulin resistance, a universal feature of type 2 diabetes mellitus. The findings of Oyadomari and colleagues suggest that critical levels of ER stress can contribute to $\beta$ cell death and the development of diabetes mellitus. The $\beta$ cell may be especially sensitive to ER stress, as mice and humans lacking PERK, a critical component of the ER stress response, develop spontaneous diabetes mellitus at a very young age (reviewed in ref. 21). Together, these findings suggest that ER stress-mediated cell death or dysfunction may play a role in the development of common forms of diabetes mellitus. It may be possible to test this hypothesis by measuring the effect of $\mathrm{CHOP}$ or Caspase-12 mutations on the progressive diabetic phenotype of $D b / D b$ (or $\mathrm{Ob} / \mathrm{Ob}$ ) C57BL/6KsJ mice. The latter provide good mouse models for the $\beta$ cell attrition in human type 2 diabetes mellitus (22).

Evidence reviewed here for the possible role of ER stress-mediated programmed cell death and dysfunction in the pathogenesis of important 
human diseases suggests that the mediators of this response may be targets for therapeutic intervention. Enthusiasm for targeting the CHOP and Caspase-12 pathways should be tempered by the realization that mutations in either gene do not fully protect against cell death caused by ER stress. The phenotype of mutations that block CHOP's upstream activators, PERK and phosphorylated eIF $2 \alpha$, reveals some of the complexities involved: $P E R K^{-1-}$ cells and cells with mutations that prevent phosphorylation of PERK's substrate, eIF2 $\alpha$, are unable to activate CHOP expression in response to ER stress $(18,23)$, yet both are dramatically hypersensitive to ER stress $(15,23,24)$. PERK phosphorylation of eIF $2 \alpha$ contributes substantially to both translational and transcriptional control in the UPR $(15,23)$. Therefore, the phenotype of the PERK and $e I F 2 \alpha$ mutations noted above demonstrates the important role of protective mechanisms against ER stress. These observations also suggest that augmenting these protective responses may be as important as blocking the known mediators of ER stress-induced cell death. In this broader context, the paper by Oyadomari and colleagues (3) should serve as a stimulus for further research into basic mechanisms of the ER stress response.

1. Hightower, L.E. 1991. Heat shock, stress proteins, chaperones, and proteotoxicity. Cell. 66:191-197.

2. Carrell, R.W., and Lomas, D.A. 1997. Conformational disease. Lancet. 350:134-138.

3. Oyadomari, S., et al. 2002. Targeted disruption of the Chop gene delays endoplasmic reticulum stress-mediated diabetes. J. Clin. Invest. 109:525-532. DOI:10.1172/JCI200214550.

4. Wang, J., et al. 1999. A mutation in the insulin 2 gene induces diabetes with severe pancreatic $\beta$ cell dysfunction in the Mody mouse. J. Clin. Invest. 103:27-37.

5. Ellgaard, L., Molinari, M., and Helenius, A. 1999. Setting the standards: quality control in the secretory pathway. Science. 286:1882-1888.

6. Leroux, L., et al. 2001. Compensatory responses in mice carrying a null mutation for Ins1 or Ins2. Diabetes. 50(Suppl. 1):S150-S153.

7. Kaufman, R.J. 1999. Stress signaling from the lumen of the endoplasmic reticulum: coordination of gene transcriptional and translational controls. Genes Dev. 13:1211-1233.

8. Mori, K. 2000. Tripartite management of unfold ed proteins in the endoplasmic reticulum. Cell. 101:451-454.

9. Bertolotti, A., Zhang, Y., Hendershot, L., Harding, H., and Ron, D. 2000. Dynamic interaction of BiP and the ER stress transducers in the unfolded protein response. Nat. Cell Biol. 2:326-332.

10. Liu, C.Y., Schroder, M., and Kaufman, R.J. 2000 Ligand-independent dimerization activates the stress-response kinases IRE1 and PERK in the lumen of the endoplasmic reticulum. J. Biol. Chem. 275:24881-24885.

11. Zinszner, H., et al. 1998. CHOP is implicated in programmed cell death in response to impaired function of the endoplasmic reticulum. Genes Dev. 12:982-995.

12. Nakagawa, T., et al. 2000. Caspase-12 mediates endoplasmic-reticulum-specific apoptosis and cytotoxicity by amyloid-beta. Nature. 403:98-103.

13. Urano, F., et al. 2000. Coupling of stress in the endoplasmic reticulum to activation of JNK protein kinases by transmembrane protein kinase IRE1. Science. 287:664-666

14. Wang, X.Z., et al. 1998. Cloning of mammalian Ire 1 reveals diversity in the ER stress responses. EMBO J. 17:5708-5717.

15. Harding, H., et al. 2001. Diabetes mellitus and excocrine pancreatic dysfunction in Perk-/- mice reveals a role for translational control in survival of secretory cells. Mol. Cell. 7:1153-1163.

16. McCullough, K.D., et al. 2001. Gadd153 sensitizes cells to endoplasmic reticulum stress by down-regulating $\mathrm{Bcl} 2$ and perturbing the cellular redox state. Mol. Cell. Biol. 21:1249-1259.

17. Fawcett, T.W., et al. 1999. Complexes containing activating transcription factor (ATF)/cAMPresponsive-element-binding protein (CREB) interact with the CCAAT/enhancer-binding protein (C/EBP)-ATF composite site to regulate Gadd153 expression during the stress response. Biochem. J. 339:135-141.

18. Harding, H., et al. 2000. Regulated translation initiation controls stress-induced gene expression in mammalian cells. Mol. Cell. 6:1099-1108.

19. Gow, A., and Lazzarini, R.A. 1996. A cellular mechanism governing the severity of PelizaeusMerzbacher disease. Nat. Genet. 13:422-428.

20. Muller, H.W. 2000. Tetraspan myelin protein PMP22 and demyelinating peripheral neuropathies: new facts and hypotheses. Glia. 29:182-185.

21. Sonenberg, N., and Newgard, C.B. 2001. Protein synthesis. The perks of balancing glucose. Science. 293:818-819.

22. Baetens, D., et al. 1978. Alteration of islet cell populations in spontaneously diabetic mice. Diabetes. 27:1-7.

23. Scheuner, D., et al. 2001. Translational control is required for the unfolded protein response and in-vivo glucose homeostasis. Mol. Cell. 7:1165-1176.

24. Harding, H., Zhang, Y., Bertolotti, A., Zeng, H., and Ron, D. 2000. Perk is essential for translational regulation and cell survival during the unfolded protein response. Mol. Cell. 5:897-904.

25. Gow, A., Southwood, C.M., and Lazzarini, R.A. 1998. Disrupted proteolipid protein trafficking results in oligodendrocyte apoptosis in an animal model of Pelizaeus-Merzbacher disease. J. Cell Biol. 140:925-934.

26. D’Urso, D., Prior, R., Greiner-Petter, R., GabreelsFesten, A.A., and Muller, H.W. 1998. Overloaded endoplasmic reticulum-Golgi compartments, a possible pathomechanism of peripheral neuropathies caused by mutations of the peripheral myelin protein PMP22. J. Neurosci. 18:731-740.

27. Ito, M., and Jameson, J.L. 1997. Molecular basis of autosomal dominant neurohypophyseal diabetes insipidus. Cellular toxicity caused by the accumulation of mutant vasopressin precursors with in the endoplasmic reticulum. J. Clin. Invest. 99:1897-1905

28. Davis, R.L., et al. 1999. Familial dementia caused by polymerization of mutant neuroserpin. Nature. 401:376-379.

29. Perlmutter, D.H. 1998. Alpha-1-antitrypsin deficiency. Semin. Liver Dis. 18:217-225.

30. Lamande, S.R., and Bateman, J.F. 1999. Procollagen folding and assembly: the role of endoplasmic reticulum enzymes and molecular chaperones. Semin. Cell Dev. Biol. 10:455-464. 